\title{
CALCIUM NECROSIS OF THE SKIN
}

\author{
BY
}

\section{A. G. HEPPLESTON}

From the Department of Pathology, Welsh National School of Medicine

A method of dust suppression recently introduced into mining depends upon the hygroscopic properties of calcium chloride, a 40 per cent. aqueous solution, to which is added 2-4 per cent. of a wetting agent (' Lissopol $\mathrm{N}$ ' I.C.I.), being used for spraying the roadways of mines. Contact with the solution was presumed to be innocuous but the following case showed that this was not true.

\section{Case Record}

J. C. W., while engaged in spraying in a South Wales coal mine, allowed some of the solution to contaminate his skin. Immediately af ter exposure, which lasted 8 hours, he experienced burning pain accompanied by the formation of 'wheals' on the upper eyelids, forearms, and thighs. Similar effects were noticed on his hands and legs, where abrasions occurred during the work, those on the legs being due to friction from the tops of his rubber boots. Eleven days later the lesions on the legs and hands broke down. When first examined 16 days after exposure there were large sloughing ulcers with raised, dark red edges running transversely across the upper parts of both legs and smaller round ones on the backs of both hands, with crusts which, when removed, left deep pits. The lesions on the eyelids, forearms, and thighs were not ulcerated but consisted of pearly yellow, raised spots measuring 1-2 mm., some of them round and others shaped like splashes.

These lesions have been observed for over 5 months. The ulcers were indolent, and epithelialization was not completed until after 13 weeks, but from the seventh week onwards those on the legs were outlined by narrow zones of yellow discolouration of the deeper tissues. The appearance of the non-ulcerated lesions did not change for the first 4 months, but they finally disappeared.

Another man who was engaged in the same work noticed burning and redness at the sites of contact immediately afțer exposure. He alleged that he then washed very thoroughly with soap and that the effects disappeared in about a week.

\section{Histology}

Biopsies were taken from lesions of the forearm, hand and leg 18 days after exposure. The essential features were shown best by one of the nonulcerated lesions from the right forearm. This exhibited a sharply circumscribed area of coagulative necrosis of the dermis, the necrotic collagen fibres being heavily impregnated with calcium salt, while the overlying epidermis was normal, apart from some loss of cellular definition. Here no inflammatory reaction could be seen (fig. 1), but in a severely ulcerated lesion from the right leg the picture was modified by inflammation. There the calcium-impregnated tissue was less conspicuous and showed a granular fragmentation and cellular infiltration. Sweat gland epithelium in the affected area was damaged in some parts but escaped in others. A small ulcer from the right hand had an intermediate appearance, there being less inflammation and more calcium necrosis.

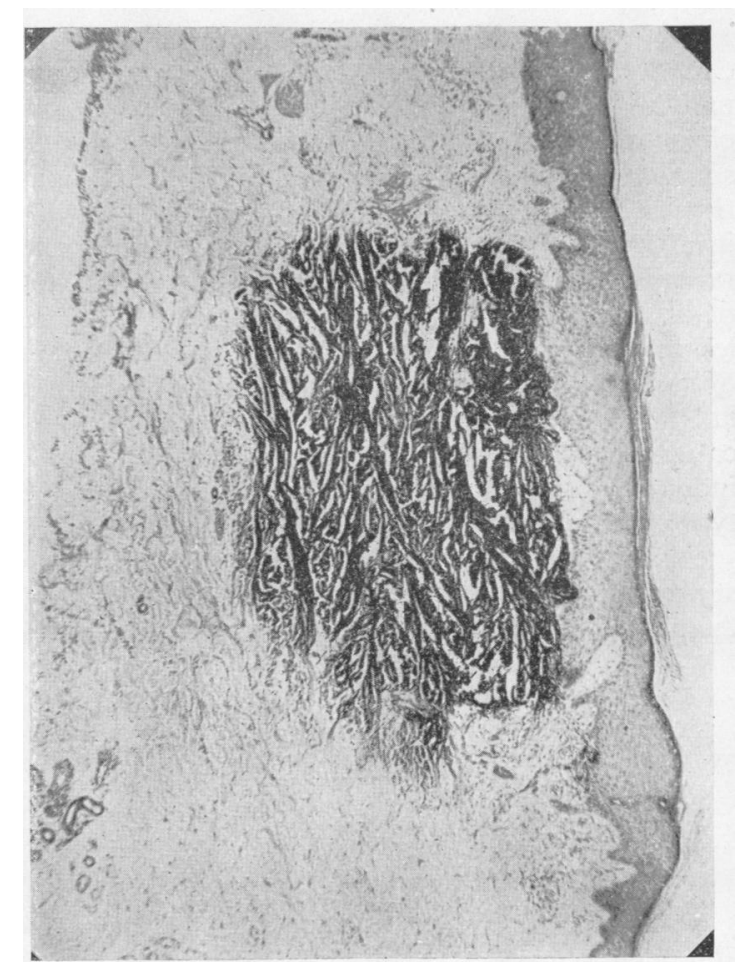

FIG. 1.-Non-ulcerated lesion, right forearm. Stained von Kossa, haematoxylin and eosin $(\times 40)$.

\section{Discussion}

The development of two types of lesion apparently depended upon whether the skin was injured or not at the time of contact with the solution. In the absence of injury, calcium infiltration with necrosis of the dermis alone developed, but where there were abrasions, inflammation and ulceration followed. The progress of the severely ulcerated lesions on the legs also indicated that complete resolution only occurred where the inflammatory reaction was sufficiently intense to effect disintegration and discharge of the dead tissue. In the centre of the ulcers the reaction was marked, and healing, though rather delayed, was satisfactory. At their edges, where it was less intense, yellow zones remained; and 
these, judging from the non-ulcerated lesions, represented persistent subepidermal deposits of necrotic material which in time may be removed without surgical intervention. It was significant that in both types of lesion healing took place most readily at the biopsy sites where the necrotic tissue was removed, showing that persistence of the latter was responsible for the delayed or incomplete resolution.

The fact that the epithelium over the nonulcerated lesion was relatively normal (had the epithelium been regenerated one would not expect it to possess rete pegs) suggested that strong calcium chloride solution by itself can penetrate the epidermis and leave only minimal histological evidence of damage.

Lesions resembling those on the hands of this case were described by Oppenheim (1935) in an individual who, during the course of work as an icecream maker, dropped concentrated calcium chloride solution on to the insteps. Histological examination could not be made, so instead Oppenheim investigated the action of calcium chloride on the rabbit's skin. Intracutaneous injection of $0 \cdot 1 \mathrm{c.cm}$. of a 10 per cent. solution evoked, after 3 weeks, lesions somewhat similar to the non-ulcerated ones observed in the present case; they differed in that an inflammatory reaction also developed. Scarification followed by the application of the solid salt induced a response comparable to the ulcerative type of human lesion. Calcium chloride was used specifically as a necrosing agent in the experimental study of silico-tuberculosis (Kettle, 1924) and of tetanus (Russell, 1927), but naturally neither of these lesions bore any histological resemblance to the human ones.

The part played by the wetting agent in the production of the lesions was uncertain. Its reputed harmlessness to normal skin when applied in a pure state suggested that a direct influence was negligible, but it may have facilitated penetration of the skin by the calcium chloride.

\section{Summary and Conclusions}

1. A case is described in which multiple cutaneous necroses developed as a result of contact with 40 per cent. calcium chloride solution.

2. The characteristic lesion was a non-ulcerated focal necrosis of the dermis without evidence of any kind of inflammatory reaction.

3. The lesions proved to be indolent and their delayed or incomplete resolution is attributed to the persistence of calcium-necrosed tissue. Where there was also inflammatory ulceration healing was assisted.

4. The use of such a solution for industrial purposes demands adequate protection of the skin and especially of the eyes.

5. Thorough and immediate washing, using plenty of soap, may mitigate the effects of contamination.

\section{Acknowledgements}

I am indebted to the patient for his co-operation and to Professor J. B. Duguid for criticism and advice. Mr. A. S. Aldis performed the biopsies and Mr. G. R. Armstrong rendered technical assistance.

\section{REFERENCES}

Kettle, E. H. (1924), Brit. J. Exp. Path., 5, 158. Oppenheim, M. (1935). Wien. klin. Wchnschr., 48, 207. 